\title{
COVID-19, social isolation, and psychological distress in a Brazilian sample
}

Raíssa Oliveira de Mendonça ${ }^{1}$, Mylena Maria Ribeiro de Almeida ${ }^{1}$, Talita Barroso Garcia ${ }^{1}$, Normando José Queiroz Viana ${ }^{1,2}$, Caio Maximino ${ }^{1,3^{*}}$

${ }^{1}$ Faculdade de Psicologia, Instituto de Estudos em Saúde e Biológicas, Universidade Federal do Sul e Sudeste do Pará

${ }^{2}$ Núcleo de Estudos Psicossociais em Saúde, Faculdade de Psicologia, Instituto de Estudos em Saúde e Biológicas, Universidade Federal do Sul e Sudeste do Pará

${ }^{3}$ Laboratório de Neurociências e Comportamento "Frederico Guilherme Graeff", Faculdade de Psicologia, Universidade Federal do Sul e Sudeste do Pará

${ }^{*}$ Corresponding author

Caio Maximino

Laboratório de Neurociências e Comportamento "Frederico Guilherme Graeff", Faculdade de Psicologia

Universidade Federal do Sul e Sudeste do Pará - Campus Marabá, Unidade III Av. dos Ipês, S/N

68500-000 - Marabá/PA, Brazil

cmaximino@unifesspa.edu.br 


\section{Abstract}

Importance: The global infection outbreak by the new SARS-CoV-2 prompted community containment schedules; however, social isolation is a predictor of psychological distress.

Objective: To determine whether social isolation, in Brazil, led to higher signs of psychological distress, and which intra- and inter-psychic variables mediated this effect. The following hypotheses were tested: 1) in isolated individuals, loneliness activates distancing and escape-avoidance coping strategies with intensities that are directly correlated with symptoms of anxiety and common health disorders; 2) in isolated individuals, poor reliance on social support coping strategies increase the effects of loneliness on symptoms of anxiety and common mental disorders; 3) in isolated individuals, External Entrapment moderates the effects of loneliness such that the higher the feelings of entrapment, the higher the effects of loneliness on symptoms of anxiety and common mental disorders. 4) in both isolated and non-isolated individuals, intense reliance on positive reappraisal coping strategies decrease (moderate) the effects of information consumption on symptoms of anxiety and common mental disorders. No a priori hypothesis on the specific nature of the subjective experiences of social isolation were established. We proposed that the semantic field of social isolation should present a complex and multidimensional nature. Design: Two phases of web-based surveys were applied to participants between May $25^{\text {th }} 2020$ and August $19^{\text {th }}$ 2020. Setting: Brazilian participants responded surveys on the web.

Participants: For Phase 1, 440 participants responded to the survey. Participants were a volunteer sample of Brazilian nationality and above 18 years old. For Phase 2, a sub-sample of 55 participants was drawn from the pool of the first phase. Main outcomes and measures: For Phase 1, the primary endpoint was score in the SRQ-20 scale (an instrument to screen symptoms of common mental disorders), and the secondary endpoint was score in an anxiety scale that screened feelings of anxiety related to illness and medical procedures. Results: For Phase 1,51\% of the sample reported leaving the house less than once a week during the period of the research, $27.6 \%$ reported leaving the house 1-2 times per week, 9.8\% reported leaving the house 3-4 times per week, and $11.6 \%$ reported leaving the house every day. Using SRQ-20, we found that $76.9 \%$ of the female respondents and $58.0 \%$ of the male respondents that reported leaving their houses less than once a week showed clinically significant symptoms, while these proportions fell below $65 \%$ for females and $44 \%$ for males that reported leaving their houses more than 3 times per week. Reliance on escape/avoidance as well as distancing coping strategies were significant mediators of the effect of isolation-induced loneliness. We did not find support for the hypothesis that 
reliance on social support coping strategies significantly altered the effects of social isolation-induced loneliness on psychological distress, nor for the hypothesis that external entrapment moderated the effects of loneliness. We also found that the impact of reliance on positive reappraisal coping strategies on the relationship between frequency of media use for COVID-19-related information and psychological distress depended on the type of media, with individuals which sought information from print or online newspapers, social networks, and podcasts at higher frequencies consistently showing more psychological distress; however, higher levels of positive reappraisal coping strategies increased this impact instead of decreasing it. In Phase 2 (qualitative survey), $47.3 \%$ of the sample reported leaving the house less than once a week during the period of the research, $21.8 \%$ reported leaving the house 1-2 times per week, $10.9 \%$ reported leaving the house $3-4$ times per week, and $20 \%$ reported leaving the house every day. At the qualitative survey we found that individuals interpreted isolation as producing self-assessment with protective and introspective dimensions, but also ruminative and emotional experiences of distress. Conclusions and relevance: Our results reveal that social isolation during the COVID-19 pandemic significantly increased psychological distress at clinically relevant rates, with loneliness being an important predictor of this effect. We also found that escape-avoidance and distancing coping strategies mediated this effect. Psychological distress was also related to high consumption of COVID-19-related information in social networks, print or online newspapers, and podcasts, but that relying on positive reappraisal coping strategies increased this effect instead of decreasing it. Our results suggest the need for policies that diminish the impact of social isolation on mental health; the need to assess and teach alternative coping strategies in clinical settings; and the need to address the impact of Internet-based sources.

Keywords: COVID-19; social isolation; mental health; coping strategies; media consumption; entrapment 
The global infection outbreak by the new SARS-CoV-2, inducing a high mortality from the associated disease (COVID-19), quickly spread to practically all countries in the world due to its rapid transmission. COVID-19 is a disease of great impact on human populations, due to its high mortality and transmission by respiratory droplets ${ }^{1}$. An important consequence of this high transmissibility, as well as the absence of vaccines or treatment protocols, is that public health actions related to COVID-19 aim to decrease the probability of encounter between infected and susceptible individuals, decreasing the rate of transmission ${ }^{1,2}$. As a rule, countries that promptly adopted community containment schemes (i.e., as soon as community transmission status of the SARS-CoV-2 coronavirus was found) have had relative success in reducing mortality and infection rate to date ${ }^{3}$.

The objective of community confinement is to reduce contact of unidentified infected individuals with non-infected members of the community ${ }^{2}$. It is particularly useful in contexts where community transmission is substantial, but involves ethico-political difficulties ${ }^{4}$ and possible impacts on mental health ${ }^{5}$. In effect, in a national survey on complaints of psychological distress in the Chinese population during and after the COVID-19 outbreak (responses collected up to February 10, 2020), about $35 \%$ of respondents showed some important sign of distress (score in COVID-19 Peritraumatic Distress Index between 28 and 51 , indicating moderate stress) ${ }^{6}$. Authors attributed this impact on the psychological functioning and well-being to three main events: 1) the official confirmation of human transmission of the SARS-CoV-2 coronavirus; 2) the strict quarantine of Wuhan; and 3) the announcement by the World Health Organization that the new coronavirus was a Public Health Emergency of International Concern. In the UK, psychiatric symptoms, as assessed by the shorter version of the General Health Questionnaire (GHQ-12), increased one month into the lockdown by 0.48 points higher than expected when accounting for previous upward trends between 2014 and $2018^{7}$. Taquet et al. ${ }^{8}$ observed that, after lockdown, mood homeostasis decreased among Dutch students, with larger decreases associated with larger decreases in mood.

There is potentially a miriad of factors that are associated with psychological distress during the COVID-19 pandemic ${ }^{5}$. Since social isolation is an important mediator of psychological distress, it is likely that community containment schemes are responsible for these effects. Another study of Chinese populations identified signs of acute stress in individuals who had practiced self-isolation for 14 days (as of January 2020), and suggested that "social capital" (a set of current or potential resources that include social confidence, sense of belonging, and participation ${ }^{9}$ ) mediates the effect of isolation on the quality of sleep, anxiety, and acute stress ${ }^{10}$. Nonetheless, in a nationwide survey of psychological distress 
symptoms in Italian people during the COVID-19 pandemic, going to work instead of staying at home was associated with higher stress levels ${ }^{11}$. These preliminary results suggest a negative impact of social isolation caused by community containment schemes, but it is also possible that the existential risk of the disease ("fear of dying" or "fear of losing a loved one") produced these effects. The Italian data showed that having a relative going to work was also a predictor of higher stress ${ }^{11}$. In the SARS outbreak that occurred in Hong Kong in 2003-2004, most respondents report symptoms of psychological distress, including symptoms of post-traumatic stress and/or acute stress, even in the absence of social isolation ${ }^{12}$.

While not the sole source of distress during a pandemic, social isolation has been shown to negatively affect psychologial well-being outside of lockdown as well. Social isolation produces various psychophysiological, cardiovascular, immune, and endocrine effects; a meta-analysis of these effects has suggested that the perception of social support is strongly correlated with lower levels of autonomic activity (e.g., lower resting blood pressure), better indicators of immunovigilance (e.g., higher levels of natural killer cell lysis), and lower levels of neurovegetative axis hormones (e.g., lower levels of urinary catecholamines $)^{13}$. The perception of social support is opposed to the feeling of loneliness, a psychological construct that describes the negative emotional response to the perception of isolation. Loneliness produces transient or chronic effects ${ }^{14}$. Short-term effects involve negative feelings, but usually motivate individuals to seek social connections ${ }^{15}$. The chronic effects, however, are associated with depression and suicide, higher rates of alcoholism, and low quality of sleep, all of which are important risk factors for mental disorder ${ }^{16}$.

Thus, understanding the relationship between the perception of loneliness and the impacts of community containment on mental health is a research priority in the area ${ }^{5}$. Some suggestions of this impact already exist; using the $\mathrm{PSCl}-16$ questionnaire, which evaluates the perception of the degree of connection with groups of relatives, neighbors, friends, colleagues and work, and more distant people ${ }^{17}$, Xiao et al. ${ }^{10}$ showed that individuals who self-isolated due to the COVID-19 outbreak in China presented sleeping difficulties, anxiety, and signs of acute stress, and that these effects were mediated by "social capital". This construct is inversely associated with the degree of perceived loneliness ${ }^{18}$, suggesting that the perception of loneliness can mediate the effects of social isolation in a pandemic situation on mental health.

Studies on loneliness suggest that its impact is indirect, reflecting differences in the ways individuals cope with the stress of daily life ${ }^{19}$. Applying the UCLA Loneliness Scale ${ }^{20}$, Cacioppo et al. ${ }^{19}$ demonstrated that individuals with low levels of perceived loneliness were 
more likely to present active coping strategies (e.g., problem solving strategies) and to seek emotional and instrumental support from others, while individuals with high levels of perceived loneliness were more likely to present passive coping strategies, including behavioral disengagement or avoidance of stressors.

Despite these findings, loneliness in a pandemic context is qualitatively different from loneliness in other contexts because it is - if community containment is successful inescapable. Thus, an important psychological construct that can mediate the effects of community containment on mental health is entrapment. Entrapment is defined as a desire to escape from an unbearable situation associated with the perception that all escape routes are blocked ${ }^{21}$. Thus, the concept of entrapment is based on theoretical models that suggest that blocking defensive behavior in the context of uncontrollability or inescapability ${ }^{22}$ induces subjective states of suffering and depression ${ }^{21}$. A sense of entrapment may be associated with stressful life events or circumstances that are particularly chronic and ongoing; however, entrapment also involves psychological processes related to the individual's subjective perception that his circumstances are uncontrollable, persevering, and inescapable ${ }^{23}$. From a psychometric point of view, entrapment scales divide the construct into Internal Entrapment (related to activation for escape motivated by aversive emotional states) and External Entrapment (when the desire to escape is stimulated by external events $)^{24}$. Entrapment was pointed as a potential moderating factor for the effects of social containment in the COVID-19 outbreak ${ }^{5}$.

Although the relationship between loneliness, social support, and psychological distress is relatively well established, little is known about how loneliness is subjectively experienced in the context of forced social isolation. The construct of loneliness admits that this is one of the possible consequences of a reduction in the quantity and quality of social interactions (i.e., small social network), and there is still ambivalence in relation to the connection between social isolation and loneliness, with inconsistent interpretations in relation to the correlates of these effects ${ }^{25-27}$. For example, in a situation of forced social isolation (such as community restraint), some individuals may refer to isolation as a catalyst for creativity, or individuals may choose to nurture significant ties with a few people, increasing the quality of those ties despite the decrease in quantity. Karnick ${ }^{28}$ suggests that the current literature on loneliness and its correlates is still unable to adequately address the lived experience of isolation; therefore, to comprehend the subjective experience of social isolation in the context of the COVID-19 pandemic in a broad way, complementary methods of in-depth qualitative research will also be needed.

Another important interpersonal factor in the negative impact of disease outbreaks is 
information consumption ${ }^{5}$. Individuals seek reliable information from traditional media or social media about pandemics, looking for brief, timely guidelines, and criticism about what to do. Information consumption can therefore be adaptive and positive for mental health; however, media reports of infectious diseases often use messages that raise risk perception, potentially amplifying public anxiety (e.g., Sell et al. ${ }^{29}$ ). In particular, social networks can be an important source of dissemination of misinformation ${ }^{30}$, amplifying perceptions of risk and uncertainty ( $\mathrm{Ng}$ et al., 2018). An example of this impact, in relation to COVID-19, was observed from a computerized sentiment analysis, applied in posts on the Chinese social network Weibo, before and after the declaration that the new coronavirus was an International Public Health Emergency ${ }^{31}$. This analysis suggested that the statement, which impacted the Weibo network in several ways, produced an increase in negative emotions (e.g., anxiety, depression, and indignation) and sensitivity to social risks ${ }^{31}$.

In addition to the acute impact of news exposure about COVID-19, repeated exposure to information on infectious diseases can exacerbate psychic suffering and stress, amplify feelings of concern, and negatively impact psychological functioning (e.g., Thompson et al. ${ }^{32}$ ). The anxiety and uncertainty produced by excessive and repeated consumption of conflicting information can lead to an increase in media consumption (in an attempt to reduce uncertainty), generating a "vicious circle" that can be difficult to break ${ }^{32,33}$. Thus, a better understanding of how repeated consumption of media relative to COVID-19 can amplify psychological distress and negatively impact mental health in various groups can help optimize patterns of information that promote well-being ${ }^{5}$.

The possible negative impact of public health strategies to contain COVID-19 on mental health is an aggravating factor that should be considered when choosing complementary policies ${ }^{5}$. A panel of researchers, supported by technical references in mental health and individuals with lived experience with mental disorders, suggested that an immediate priority for research in the area is to collect high quality data on the mental health impacts of the COVID-19 pandemic on the general population, vulnerable groups, and SARS-CoV-2 infected patients ${ }^{5}$, research that should, above all, give voice to the participants in order to raise, in an emic way, the acts of meaning that are present in the way they think about physical isolation ${ }^{34}$. Among these immediate priority objectives, the identification of causal mechanisms associated with the effects on mental health, including loneliness, sense of entrapment, coping strategies, and repeated consumption of information derived from the media, is fundamental to the creation of complementary mental health policies that reduce the impact of social isolation while maintaining the effectiveness of community confinement ${ }^{5}$. Thus, understanding the intra- and inter-psychic mechanisms that 
provide appropriate or inappropriate responses from a mental health perspective can positively impact the creation of policies and interventions that mitigate the negative effects of community confinement.

\section{Methods}

\subsection{Open science practices}

Study hypothesis, methods, and statistical analysis were preregistered on Open Science Framework (https://osf.io/g6j8n). Public access to the preregistration was opened on June $20^{\text {th }}$ 2020. Results and analysis scripts were also posted in the same platform (https://osf.io/fv7ax/).

\subsection{Participants}

For Phase 1, high-coverage population list-based samples ${ }^{35}$ were obtained by distributing the online form (see 2.3.3, below) through email lists, directed messages using instant messaging apps (WhatsApp and Telegram), social media posts (on Facebook, Instagram, Twitter, and Mastodon), and press releases sent to local newspapers. For Phase 2, an authorization was produced in the debriefing session of the survey form of Phase 1 , and responders who agreed to participate in further stages of the research were contacted by email. For the first phase (quantitative survey) of the research, 440 respondents answered an online survey on socio-demographical characteristics, social isolation practices, and media consumption habits, and responded to scales on loneliness, symptoms, disease anxiety, coping strategies, and entrapment (2.3.3, below). Sample characteristics can be found on Table 1.

In the preregistered version of this project, sample size needed to achieve $80 \%$ power for the first phase was 562 participants (https://osf.io/g6j8n). However, since community containment schemes in Brazil rapidly declined, with average isolation indexes consistently falling below $50 \%$ after July $5^{\text {th }}$ (https://mapabrasileirodacovid.inloco.com.br/pt/), we opted to stop collecting data before reaching the final sample size so as to guarantee that most respondents still were in a context of social isolation. A posteriori power was calculated using a $\mathrm{R}$ shiny script ${ }^{36}$, using hypothesis 1 , based on the analysis of the principal endpoint, 
with the sample size used in the research. Calculated power was 0.73.

For the second phase of the study (qualitative phase), a sub-sample of participants was drawn from the pool of the first phase. After answering all questions of the first phase, participants could authorize further contact for this stage. Sample characteristics for this phase can be found on Supplementary Table 1. In the preregistered version of this project, sample size projected to reach saturation of categories was based on theme accumulation curves ${ }^{37}$, with an initial sample of 50 participants proposed for drawing a interim theme accumulation curve; if the local slope of the curve is above 0.05 , another 50 participants would be recruited, and the process was re-analyzed until saturation was reached. Again, since community containment schemes in Brazil began to decline, we opted to stop collecting data, and ended with 55 respondents. The local slope of the theme accumulation curve fell consistently below 0.05 after 33 participants, suggesting that the sample size was more than enough to reach saturation (Supplementary Figure 1).

\subsection{Phase 1: Quantitative survey}

\subsubsection{Hypotheses}

Higher loneliness levels have been associated with higher reliance on passive coping strategies, including behavioral distancing and stressor avoidance, in the general population 19. Thus, the first hypothesis that was tested in this work was that in isolated individuals, loneliness activates distancing and escape-avoidance coping strategies with intensities that are directly correlated with symptoms of anxiety and common health disorders.

Seeking social support can also be related to how feelings of loneliness are associated with distress in isolated individuals ${ }^{10}$. The second hypothesis that was tested in this work was that, in isolated individuals, poor reliance on social support coping strategies increase the effects of loneliness on symptoms of anxiety and common mental disorders.

Since loneliness, under community confinement, is mostly imposed from the outside, its effects might be mediated by a sense of External Entrapment ${ }^{21}$. The third hypothesis that was tested in this work was that, in isolated individuals, External Entrapment moderates the effects of loneliness such that the higher the feelings of entrapment, the higher the effects of loneliness on symptoms of anxiety and common mental disorders.

Information consumption is an important factor in psychological distress during pandemics and disasters ${ }^{29,32}$. While media information can lead to distress, it can also lead to the use of 
positive reappraisal strategies that can moderate the effects of higher media consumption. Thus, the fourth hypothesis that was tested in this work was that in both isolated and nonisolated individuals, intense reliance on positive reappraisal coping strategies decrease (moderate) the effects of information consumption on symptoms of anxiety and common mental disorders.

\subsubsection{Survey instruments}

For this quantitative survey phase, participants responded to an online form on deGoogle Forms. The form presented a socio-economic questionnaire, and its results are presented on Supplementary Table 1. The form also presented two questions on isolation practices: "How often have you been leaving your house per week?" and "If you answered 3-4 times per week or Everyday, did you leave the house because you were working?". The first question was used to construct four levels of isolation ("Less than once per week", "1-2 times per week", "3-4 times per week", and "Every day").

Primary (common mental disorders symptoms) and secondary (anxiety symptoms) endpoints were assessed through responses to the Self-Report Questionnaire 20 (SRQ-20), an instrument that is used to screen symptoms of common mental disorders ${ }^{38}$, and through the Outpatient and Hospital Patients Anxiety Scale, and instrument that is used to quantify degree of intensity regarding physical and psychological symptoms related to anxiety and illness ${ }^{39}$.

The covariates and mediators were measured using well-established scales and instruments. Loneliness was measured using the Brazilian adaptation of the UCLA Loneliness Scale ${ }^{40}$. Coping strategies were assessed through the Brazilian adaptation of the Folkman and Lazarus Ways of Coping Questionnaire ${ }^{41}$. Feelings of external entrapment were analyzed using the Portuguese adaptation of the Entrapment Scale ${ }^{24}$. Sources of information were investigated using a inventory of sources, with a question asking the frequency (less thank once per week; 1-3 times per week; more than 4 times per week; once per day; 2-3 times per day; more than 4 times per day) that the respondent consulted one of seven information sources on updates on the COVID-19 pandemic (Radio; Television; Printed or online newspapers; Twitter, Facebook, or Instagram; Whatsapp and other instant messengers; Youtube videos; and/or Podcasts).

Individuals were not required to answer all questions; therefore, in some individuals missing values were present. As an a priori criteria for exclusion, if more than $25 \%$ of the values for a single instrument or scale were missing for one participant, all data from that participant was to be excluded; however, that did not happen for any participant. We also did not detect 
random responding (using infrequency scales with error balancing thresholds ${ }^{42}$ ).

\subsubsection{Data analysis}

To analyze quantitative data with mediation models, we used GLM mediation models based on Baron and Kenny's causal steps method ${ }^{43}$. For Hypotheses 1 and 2, the model was applied using isolation levels as factor, loneliness as co-variate, and scores on either the Escape/Avoidance, the Distancing, or the Social Support subscales of the Ways of Coping Questionnaire as mediator. For Hypothesis 3, the mediator was score on the External Entrapment subscale of the Entraptment Scale. For Hypothesis 4, each of the media sources (Radio; Television; Printed or online newspapers; Twitter, Facebook, or Instagram; Whatsapp and other instant messengers; Youtube videos; and/or Podcasts) was assessed independently, with frequency of use as factor, and scores in the Positive Reappraisal subscale of the Ways of Coping Questionnaire as moderator. In all situations, factors were coded with simple contrasts, and covariates were scaled by centering around mean \pm 1 standard deviation. Estimates are reported with 95\% confidence intervals around the estimate. All models were run using the jamovi module 'jAMM' ${ }^{44}$.

\subsection{Phase 2: Qualitative survey}

\subsubsection{Hypothesis}

No a priori hypothesis on the specific nature of the subjective experiences of social isolation were established. We proposed that the semantic field of social isolation should present a complex and multidimensional nature; therefore, while methods and analysis strategies were preregistered, results from this phase should be seen as exploratory in nature.

\subsubsection{Cognitive free writing task}

After answering the socio-economic survey, participants were asked to write freely on a question prompt: "What comes to your mind when you think about physical isolation?" Participants had no time or word limit to answer to the prompt. 


\subsubsection{Data analysis}

Data were analyzed using Thematic Content Analysis ${ }^{45}$. Thematic content analysis is a typology of the classic Bardin content analysis, widely used in qualitative research in Social Psychology, as well as in the human and social sciences. From the participants' textual production, the units of analysis were identified, their frequencies checked, and those that saturated the most were identified and organized into thematic categories, which were later conceptualized and presented.

\subsection{Ethical note}

Participants signed online informed consent forms before data collection begun. The project was cleared by an Institutional Review Board (Universidade Federal do Pará, Instituto de Ciências da Saúde), under opinion \#4.042.090. A version of the opinion in Portuguese can be found at https://osf.io/zdb69/

\section{Results}

\subsection{Quantitative survey}

\subsubsection{Loneliness, social isolation, and coping strategies}

The descriptive analysis, presented in Table 1, reveals a number of differences between social isolation levels regarding the outcomes (SRQ-20 and anxiety scores), the covariate (loneliness), and the mediators (coping strategies, entrapment). There appears to be a steep decline in SRQ-20 scores, with individuals that left the house 3-4 times per week or every day showing lower scores than individuals that left the house less than once per week. Social isolation did not appear to change anxiety, nor reliance on specific coping strategies. A steep decline in loneliness scores were also observed, again with individuals that left the house 3-4 times per week or every day showing lower scores than individuals that left the house less than once per week. However, social isolation appears to change feelings of external entrapment, with participants that left the house everyday showing lower scores than participants that left the house less than once per week. 
Table 1: Summary statistics for variables used in the model by frequency of leaving the house during the COVID-19 pandemic and by media consumption

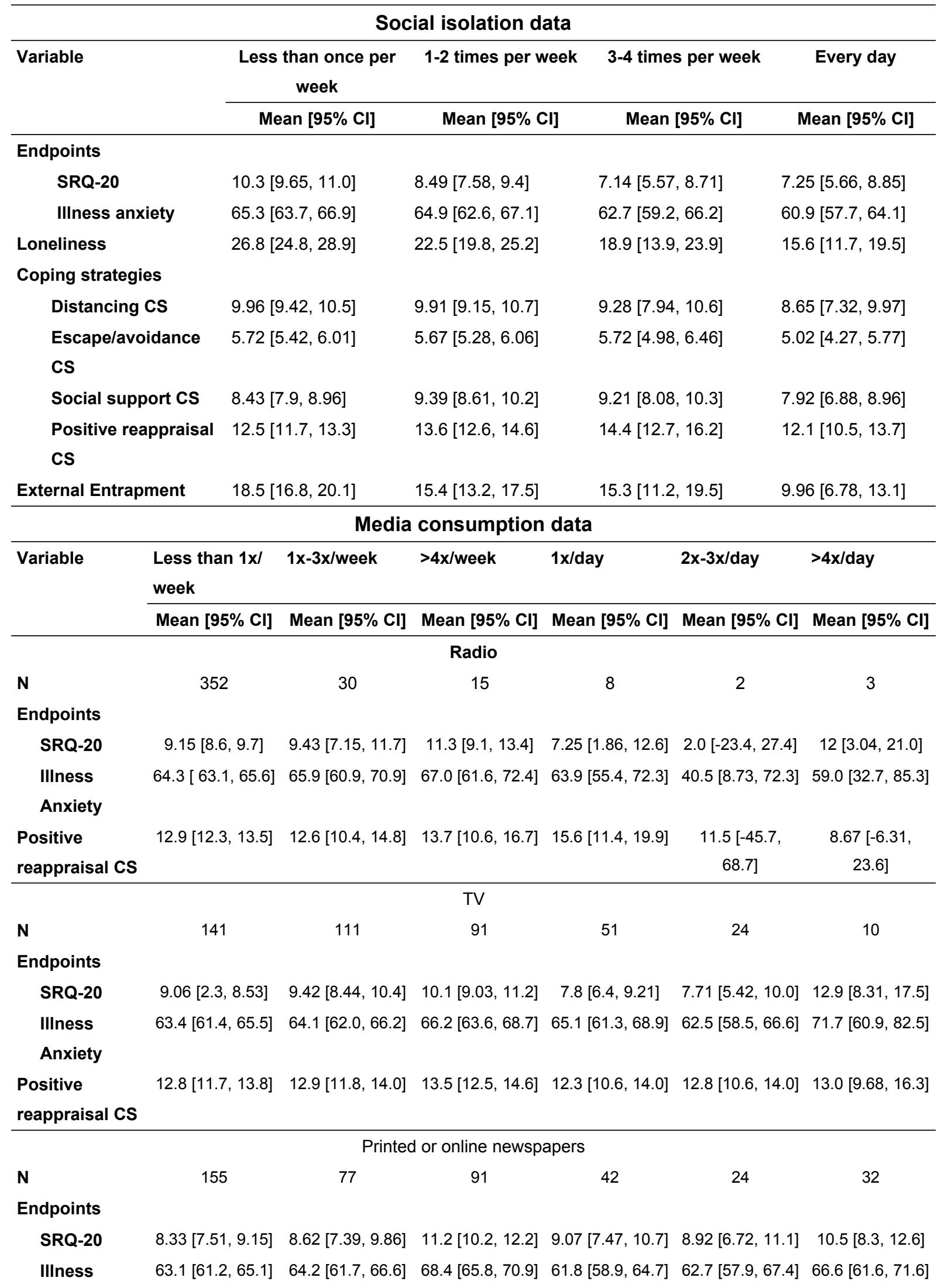




\begin{tabular}{|c|c|c|c|c|c|c|}
\hline \multicolumn{7}{|l|}{ Anxiety } \\
\hline $\begin{array}{l}\text { Positive } \\
\text { reappraisal CS }\end{array}$ & $13.2[12.3,14.0]$ & $12.8[11.5,14.2]$ & $13.3[12.1,14.5]$ & $11.8[9.92,13.7]$ & $11.5[9.08,14.0]$ & $13.4[11.4,15.5]$ \\
\hline \multicolumn{7}{|c|}{ Social networks (Facebook, Twitter, Instagram) } \\
\hline $\mathbf{N}$ & 56 & 80 & 126 & 61 & 34 & 70 \\
\hline \multicolumn{7}{|l|}{ Endpoints } \\
\hline SRQ-20 & $7.38[5.87,8.88]$ & $8.61[7.48,9.74]$ & $10.6[9.74,11.5]$ & $8.28[6.88,9.68]$ & $7.79[5.99,9.6]$ & $10.9[9.79,11.5]$ \\
\hline IIIness & $60.4[57.3,63.4]$ & $65.9[63.0,68.8]$ & $66.3[64.3,68.4]$ & $63.2[60.4,66.0]$ & $60.7[56.7,64.8]$ & $67.4[64.5,70.3]$ \\
\hline \multicolumn{7}{|l|}{ Anxiety } \\
\hline $\begin{array}{l}\text { Positive } \\
\text { reappraisal CS }\end{array}$ & $13.4[11.9,14.8]$ & $13.9[12.7,15.1]$ & $13.1[12.1,14.2]$ & $13.6[12.2,15.0]$ & $10.4[8.08,12.7]$ & $12.5[11.1,13.8]$ \\
\hline \multicolumn{7}{|c|}{ Instant messengers (WhatsApp, Telegram) } \\
\hline $\mathbf{N}$ & 123 & 78 & 105 & 46 & 19 & 53 \\
\hline \multicolumn{7}{|l|}{ Endpoints } \\
\hline SRQ-20 & $9.33[8.41,10.3]$ & $8.51[7.25,9.78]$ & $10.0[9.08,11.0]$ & $8.15[6.47,9.84]$ & $7.47[5.08,9.87]$ & $10.3[8.79,11.8]$ \\
\hline IIIness & $62.2[60.2,64.2]$ & $63.7[60.9,66.4]$ & $67.1[64.7,69.5]$ & $64.1[60.9,67.4]$ & $62.5[57.1,67.8]$ & $68.3[64.7,71.9]$ \\
\hline \multicolumn{7}{|l|}{ Anxiety } \\
\hline $\begin{array}{l}\text { Positive } \\
\text { reappraisal CS }\end{array}$ & $11.5[10.5,12.4]$ & $13.8[12.5,15.1]$ & $13.5[12.0,14.9]$ & $13.6[11.8,15.4]$ & $12.4[9.76,15.1]$ & $13.6[12.5,14.9]$ \\
\hline \multicolumn{7}{|c|}{ YouTube videos } \\
\hline $\mathbf{N}$ & 223 & 59 & 63 & 36 & 13 & 18 \\
\hline \multicolumn{7}{|l|}{ Endpoints } \\
\hline SRQ-20 & $9.07[8.38,9.77]$ & $10.0[8.63,11.4]$ & $9.62[8.39,10.9]$ & $7.89[6.13,9.65]$ & $8.62[4.75,12.5]$ & $11.4[9.02,13.8]$ \\
\hline Illness & $63.7[62.3,65.2]$ & $67.5[64.1,70.8]$ & $63.2[60.4,66.0]$ & $63.5[59.3,67.7]$ & $60.7[54.5,66.9]$ & $72.9[65.6,80.3]$ \\
\hline \multicolumn{7}{|l|}{ Anxiety } \\
\hline $\begin{array}{l}\text { Positive } \\
\text { reappraisal CS }\end{array}$ & $13.0[12.2,13.7]$ & $11.5[9.99,13.1]$ & $13.4[12.0,14.7]$ & $13.5[11.5,15.6]$ & $13.0[9.15,16.9]$ & $14.3[11.5,17.1]$ \\
\hline \multicolumn{7}{|c|}{ Podcasts } \\
\hline $\mathbf{N}$ & 302 & 48 & 30 & 14 & 5 & 5 \\
\hline \multicolumn{7}{|l|}{ Endpoints } \\
\hline SRQ-20 & $9.03[8.46,9.61]$ & $10.0[8.38,11.7]$ & $10.3[8.01,12.5]$ & $8.0[5.65,10.4]$ & $15.4[13.7,17.1]$ & $13.0[5.3,20.7]$ \\
\hline $\begin{array}{l}\text { Illness } \\
\text { Anxiety }\end{array}$ & $64.0[62.7,65.2]$ & $65.2[61.3,69.1]$ & $65.8[61.6,70.1]$ & $61.4[56.3,66.6]$ & $\begin{array}{c}83.0[58.0 \\
108.0]\end{array}$ & $75.6[55.5,95.7]$ \\
\hline $\begin{array}{l}\text { Positive } \\
\text { reappraisal CS }\end{array}$ & $13.1[12.5,13.8]$ & $12.1[10.5,13.8]$ & $12.7[10.5,14.9]$ & $11.4[7.99,14.7]$ & $15.8[6.92,24.7]$ & $11.6[3.48,19.7]$ \\
\hline
\end{tabular}

Notes: CS = coping strategy

Total sample size for each media type does not add to the final sample size due to missing data.

The relationship between loneliness and SRQ-20 scores was mediated by escape/avoidance coping depending on the level of social isolation. As Figure 1A illustrates, for the contrast between individuals who left their houses $1 x-2 x /$ week vs. those who left their houses less than once per week ("social isolation 1"), as well as for the contrast between individuals who left their houses their houses $3 x-4 x /$ week vs. those who left their houses less 
than once per week ("social isolation 2"), the effect of leaving the house on SRQ-20 was significant and negative. However, the contrast between individuals who left their houses every day vs. those who left their houses less than once per week ("social isolation 3"), this direct effect was not significant. Table 3 presents indirect effects, which suggest that, when compared with individuals leaving the house every day, for individuals in greater isolation, the relationship between isolation and SRQ-20 symptoms was mediated by loneliness and by reliance on escape/avoidance coping strategies. Effect sizes were relatively small, however: for each "step down" in social isolation, anxiety decreases by 0.06 to 0.16 points in the scale when loneliness and escape/avoidance coping strategies are accounted for.

The relationship between loneliness and anxiety scores was also mediated by escape/avoidance coping depending on the level of social isolation. As Figure 1B illustrates,, none of the direct effects were significant. Supplementary Table 2 presents indirect effects, which suggest that, for individuals in greater isolation, when compared to all other levels, the relationship between isolation and anxiety symptoms was mediated by loneliness and by reliance on escape/avoidance coping strategies. Again, effect sizes were relatively small: for each "step down" in social isolation, anxiety decreases by 0.2 to 0.5 points in the scale. 


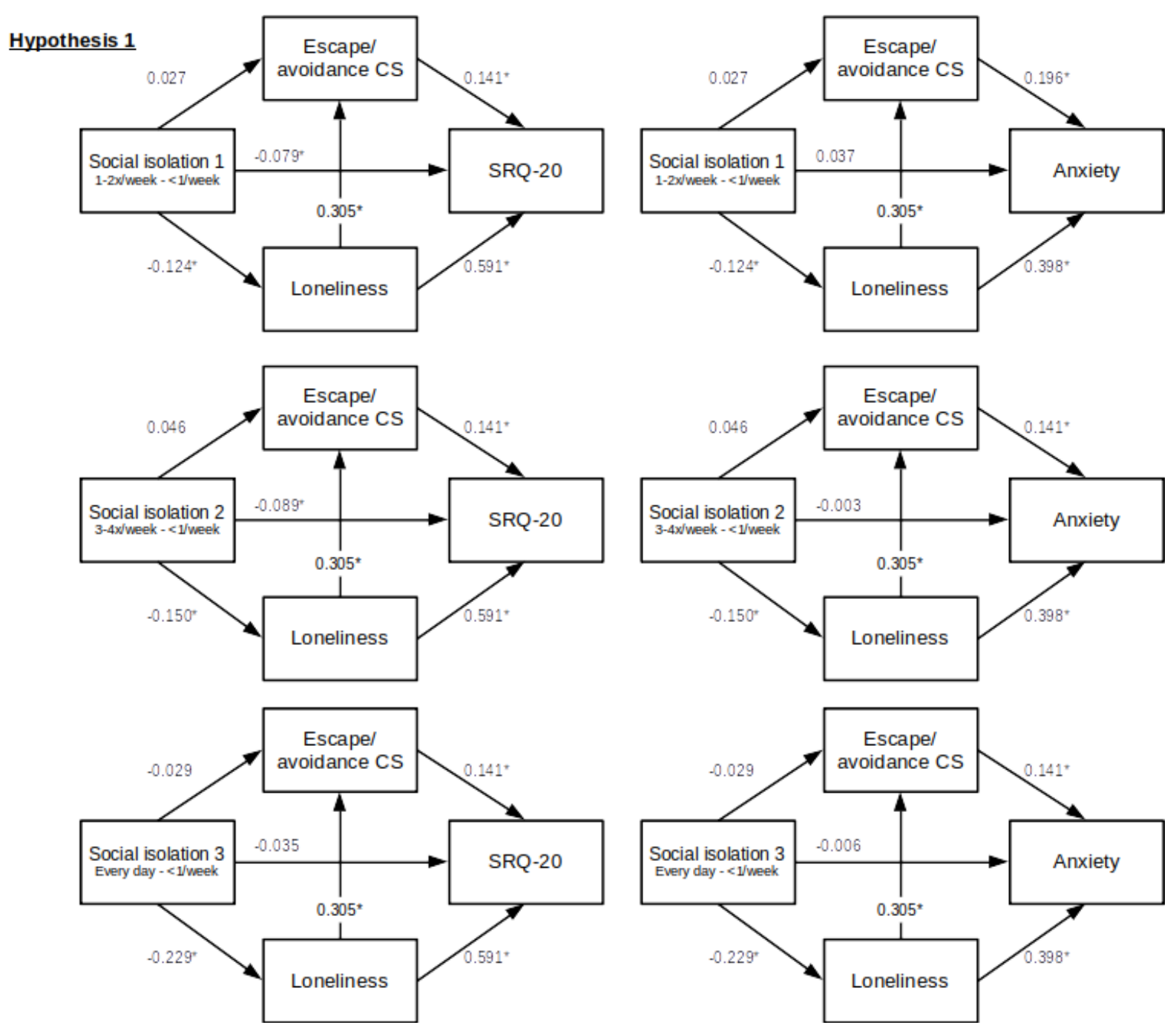

Figure 1: Standardized regression coefficients $\beta$ for the relationship between social isolation contrasts and SRQ-20 (A) or illness anxiety (B) symptoms as mediated by escape/avoidance coping strategies and loneliness.

Social isolation 1 = contrast between individuals who left their houses $1 x-2 x /$ week vs individuals who left their house $<1 x /$ week. Social isolation 2 = contrast between individuals who left their house $3 x-4 x /$ week vs individuals who left their house $<1 x /$ week. Social isolation $3=$ contrast between individuals who left their house every day vs individuals who left their house $<1 x /$ week.

${ }^{*} p<0.05$

The relationship between loneliness and SRQ-20 scores was mediated by escape/avoidance coping depending on the level of social isolation. As Figure 1A illustrates, for contrasts social isolation 1 and social isolation 2, the effect of leaving the house on SRQ20 was significant and negative. However, the contrast between individuals who left their houses every day vs. those who left their houses less than once per week ("social isolation 3 "), this direct effect was not significant. Supplementary Table 3 presents indirect effects, 
which suggest that, in contrasts social isolation 2 and social isolation 3, for individuals in greater isolation, the relationship between isolation and SRQ-20 symptoms was mediated by loneliness and by reliance on distancing coping strategies. Effect sizes were relatively small, however: for each "step down" in social isolation, anxiety decreases by 0.11 to 0.15 points in the scale when loneliness and distancing coping strategies are accounted for.

The relationship between loneliness and anxiety scores was also mediated by distancing coping strategies depending on the level of social isolation. As Figure 2B illustrates, none of the direct effects were significant. Supplementary Table 4 presents indirect effects, which suggest that, for individuals in greater isolation, when compared to all other levels, the relationship between isolation and anxiety symptoms was mediated by loneliness and by reliance on distacing coping strategies. Again, effect sizes were relatively small: for each "step down" in social isolation, anxiety decreases by 0.23 to 0.59 points in the scale. 


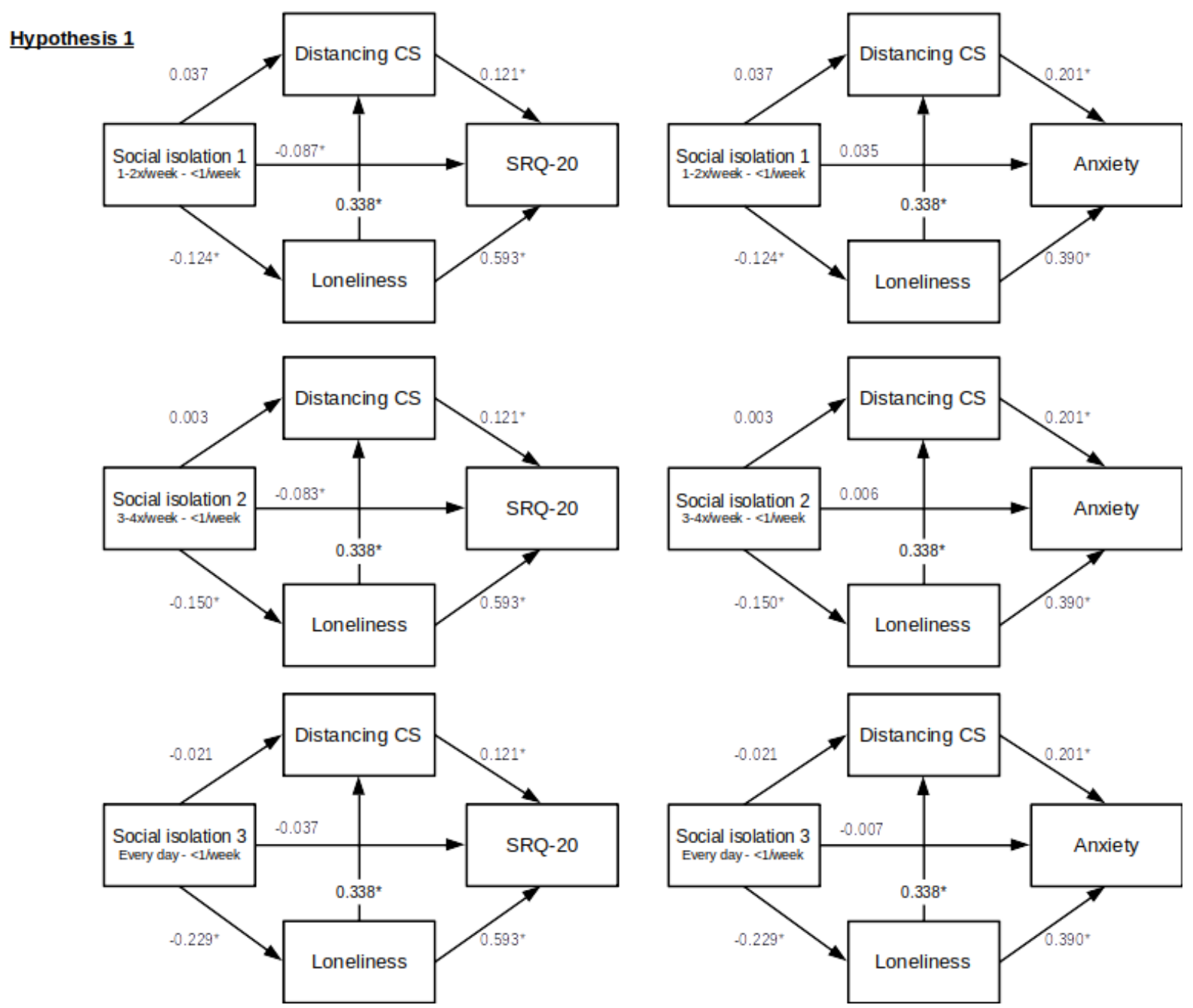

Figure 2: Standardized regression coefficients $\beta$ for the relationship between social isolation contrasts and SRQ-20 (A) or illness anxiety (B) symptoms as mediated by distancing coping strategies and loneliness. Social isolation $1=$ contrast between individuals who left their houses $1 x-2 x /$ week vs individuals who left their house $<1 \times /$ week.

Social isolation 2 = contrast between individuals who left their house $3 x-4 x /$ week vs individuals who left their house $<1 x /$ week. Social isolation $3=$ contrast between individuals who left their house every day vs individuals who left their house $<1 x /$ week.

${ }^{*} p<0.05$

Reliance on social support does not appear to impact the effect of social isolation on SRQ20 symptoms. As Figure 3 illustrates, while in contrast social isolation 1 isolation was a predictor of reliance on social support coping strategies (with individuals which left the house $1 \mathrm{x}-2 \mathrm{x} /$ week relying more on seeking social support than individuals which left the house less than once per week), this relationship was not significant at other isolation contrasts. Moreover, reliance on social support coping strategies did not predict loneliness levels. 
However, at all levels of the social isolation contrasts, reliance on social support predicted SRQ-20 symptoms (Figure 3A), with individuals that sought more social support showing more symptoms (a change of one point in the social support sub-scale indicating a change of 0.1833 point in the SRQ-20 score). The direct effects of the first two social isolation contrasts ("social isolation 1" and "social isolation 2") were also statistically significant, while indirect effects were not (Supplementary Table 4).

A similar pattern was observed with illness anxiety (Figure 3B). While both mediators were significantly associated with higher anxiety, both direct (Figure 3B) and indirect (Supplementary Table 4) effects were not statistically significant. 


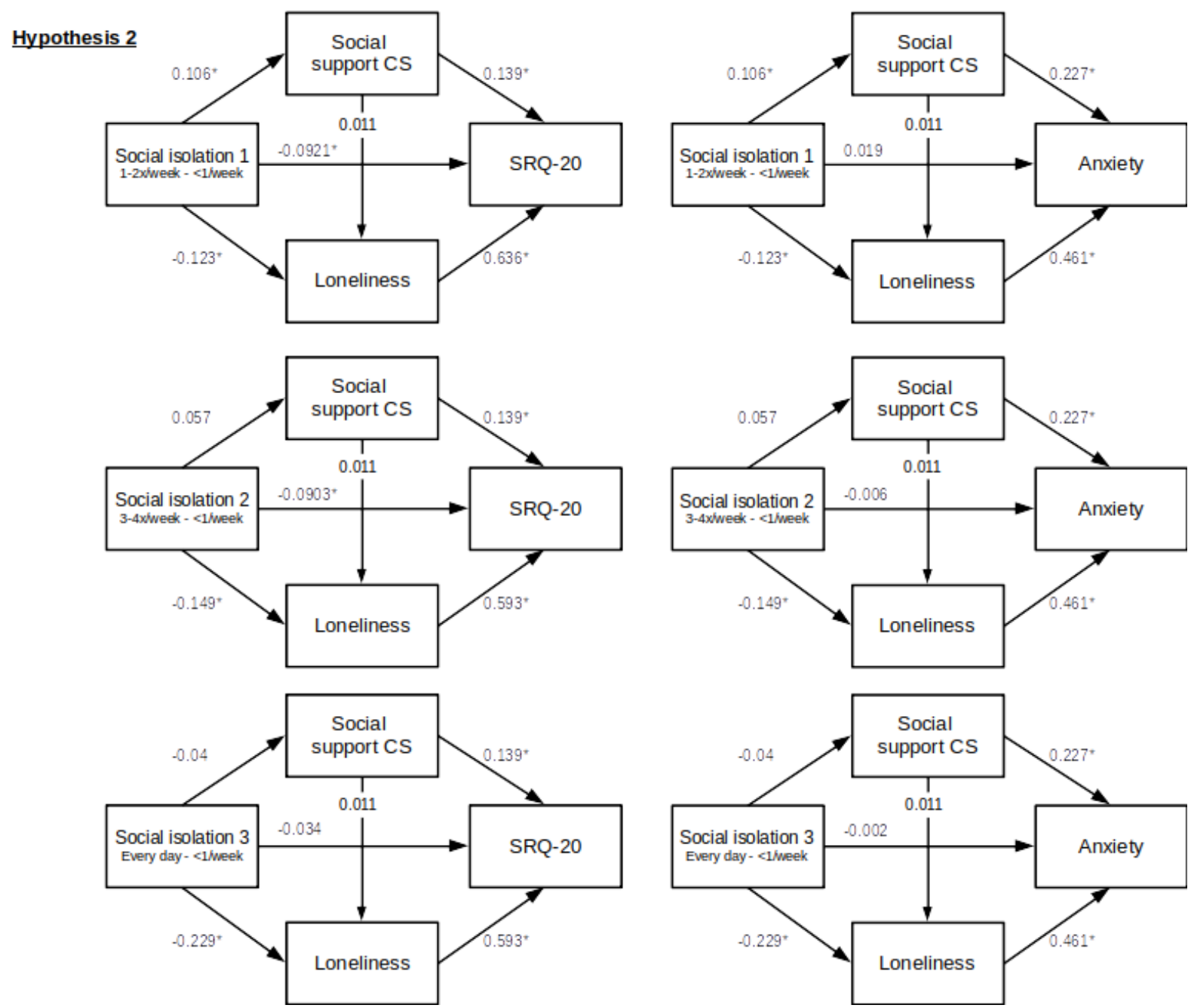

Figure 3: Standardized regression coefficients $\beta$ for the relationship between social isolation contrasts and SRQ-20 (A) or illness anxiety (B) symptoms as mediated by social support coping strategies and loneliness. Social isolation $1=$ contrast between individuals who left their houses $1 x-2 x /$ week vs individuals who left their house $<1 \times /$ week.

Social isolation 2 = contrast between individuals who left their house $3 x-4 x /$ week vs individuals who left their house $<1 x /$ week. Social isolation $3=$ contrast between individuals who left their house every day vs individuals who left their house $<1 x /$ week.

${ }^{*} p<0.05$

\subsubsection{Loneliness, social isolation, and external entrapment}

External entrapment was examined as a moderator of the relation between social isolationinduced loneliness and SRQ-20 scores. Loneliness and external entrapment were entered as the first step in the regression analysis. In the second step, the interaction term between external entrapment and loneliness was entered, and it explained a significant increase in 
SRQ-20 scores $\left(\Delta R^{2}=0.609, \chi^{2}=17.4, p<0.001\right)$. Thus, external entrapment appears to be a significant moderator in the relationship between loneliness and SRQ-20 symptoms. However, simple effects suggest that slopes did not significantly vary across levels of social isolation (Supplementary Table 5). Moreover, a 1 S.D. increase in external entrapment weakened the relationship between loneliness and SRQ-20 scores, contrary to what we hypothesized.

Similarly, when we examined external entrapment as a moderator of the relation between social-isolation induced loneliness and illness anxiety, slopes did not significantly vary across levels of social isolation nor across levels of external entrapment (Supplementary Table 5). The interaction term did not significantly explained a significant increase in illness anxiety scores $\left(\Delta R^{2}=0.975, X^{2}=0.00418, p=0.948\right)$. Thus, external entrapment does not moderate the effects of social isolation-induced loneliness and illness anxiety scores.

\subsubsection{Patterns of media consumption}

Descriptive statistics for each type of media can be found on Table 1. The table suggests that, for "traditional" media (radio, TV, printed or online newspapers), frequency of consumption of COVID-19-related information does not appear to modulate psychological distress. However, for social media (Facebook, Twitter, Instagram), the higher the frequency of looking or consuming information on COVID-19, the higher the psychological distress; the reliance on positive reappraisal coping strategies did not vary with frequency. The frequency of instant messenger use for COVID-19-related information did not appear to be related to SRQ-20 score, but frequency-dependent increases in illness anxiety and positive reappraisal were apparent. Increased frequency of YouTube videos or podcasts for COVID-19-related information appeared to increase illness anxiety, but not SRQ-20 scores or positive reappraisal.

Reliance on positive reappraisal coping strategies was examined as a moderator of the relation between frequency of consumption of information related to COVID-19 and SRQ-20 scores or illness anxiety scores. Frequency of media (radio, TV, print or online newspapers, social networks, instant messengers, Youtube, podcasts) information and positive reappraisal coping strategies were entered as the first step in the regression analysis. In the second step, the interaction term between these variables was entered. Separate tests were made for each of the information sources. Slopes did not significantly differ from zero at any level of the moderator for radio or TV for neither endpoint (Supplementary Table 6); in both cases, frequency of media use was not a significant predictor of SRQ-20 scores nor illness anxiety. Slopes significantly differed from zero at the mean or 1 S.D. below the mean of 
positive reappraisal, but not for the high level (1 S.D. above the mean), for newspapers and social networks for SRQ-20, but not illness anxiety; in both cases frequency of media use was a significant and positive predictor of SRQ-20 scores. Slopes did not differ from zero at any level of the moderator for instant messengers as predictors of SRQ-20 scores, but a significant and positive relationwith illness anxiety scores was found at low (1 S.D. below the mean) and average (mean) values of IM use frequency. Slopes did not differ from zero at any level of the moderator for Youtube videos as predictors of SRQ-20 and illness anxiety scores. Finally, slopes significantly differed from zero at the mean of positive reappraisal for SRQ-20, but not for low (1 S. D. below the mean) or high (1 S.D. above the mean), for individuals seeking information from podcasts; for illness anxiety, slopes significantly differed from zero at the average and high levels of podcasts use frequency.

\subsection{Qualitative survey}

The qualitative survey data, when subjected to the Thematic Content Analysis model ${ }^{45}$, suggested the emergence of emic meanings that point to three types of experience, namely: reflective, ruminative and emotional (Figure 4). The category that saturated the most was "Reflective Experiences", which comprises a set of self-focusing experiences of a purposeful character, when experiencing physical isolation during the pandemic. This category is made up of two subcategories, Protective Reflective Experiences and Introspective Reflective Experiences. The first of them reveals an emic repertoire characterized by ideas that isolation is "necessary", has a "preventive" character, generates "security", and lends itself to "reducing the risk of contagion" and to "protection of the community". However, it is worth noting that, even in a less expressive way, the nature of this experience also mobilized a repertoire of sense and meaning that points to an introspection movement caused by physical isolation, as can be seen in the subcategory Introspective Reflective Experiences, represented by units of analysis "introspection", "moment of meditation", "moment of reflection", and "giving some time to myself".

However, the data revealed that the subjective experiences lived during the physical isolation have also had a tense and anxious impact on the self, which is evident when the category "Ruminative Experiences" emerges, which consists of four subcategories: isolation; absence of the other, anxiety, and formality. The Isolation subcategory refers to a repertoire of meaning that describes the act of being and feeling alone, without contact with other people, having limited one's ability to come and go, which can be perceived by the units of analysis "being alone", "being islanded", "being stuck", and "not having my freedom". 
Absence of the Other is the subcategory that brings together a repertoire with meanings that point to how longing for relationships and face-to-face encounters with other people has resulted in the respondents' lives, which can be perceived through the following units of analysis: "lack of human warmth", "lack of coexistence", "lack of demonstration of affection" and "lack of face-to-face contact". We identified the subcategory Anxiety by joining expressions such as "anxiety", "worry", "stress", "increased anxiety attacks", and "restlessness"; this subcategory integrates a meaning that points to signs of the presence of psychological distress or worsening of existing poor mental health conditions. The last subcategory of this groups was called Formality, and refers to the collection of expressions that allude to a sense of compliance with the norm, which is not interpreted as very important and superficial, as can be observed in the following units of analysis: "mere formality", "does not mean much", and "to put a make up in the situation".

Less expressive, but also noticeable, was the presence of emotional elements when the respondents referred to the physical isolation caused by the SARS-CoV 2 pandemic. The emergence of the category "Emotional Experiences" reveals a repertoire of meaning present in subjective experiences with both positive and negative factor loadings. When observing the subcategory Positive Factor Load, it appears that it expresses a sense of relief, which can be observed by an analogous unit of analysis. In turn, more significant was the subjective experience marked emotional elements of negative gradient - the subcategory Negative Factor Load -, which caused a more tense and anxious experience, from the emotional and sentimental point of view, of physical isolation, which can be perceived in the units of analysis "discouragement", "tiredness", "anguish", "boredom" and "loneliness". It is also worth noting that, in addition to the emotional experiences that are more easily identified and named, another one of a less delineated nature that is difficult to symbolize was also identified, represented by the "strange" unit of analysis. 


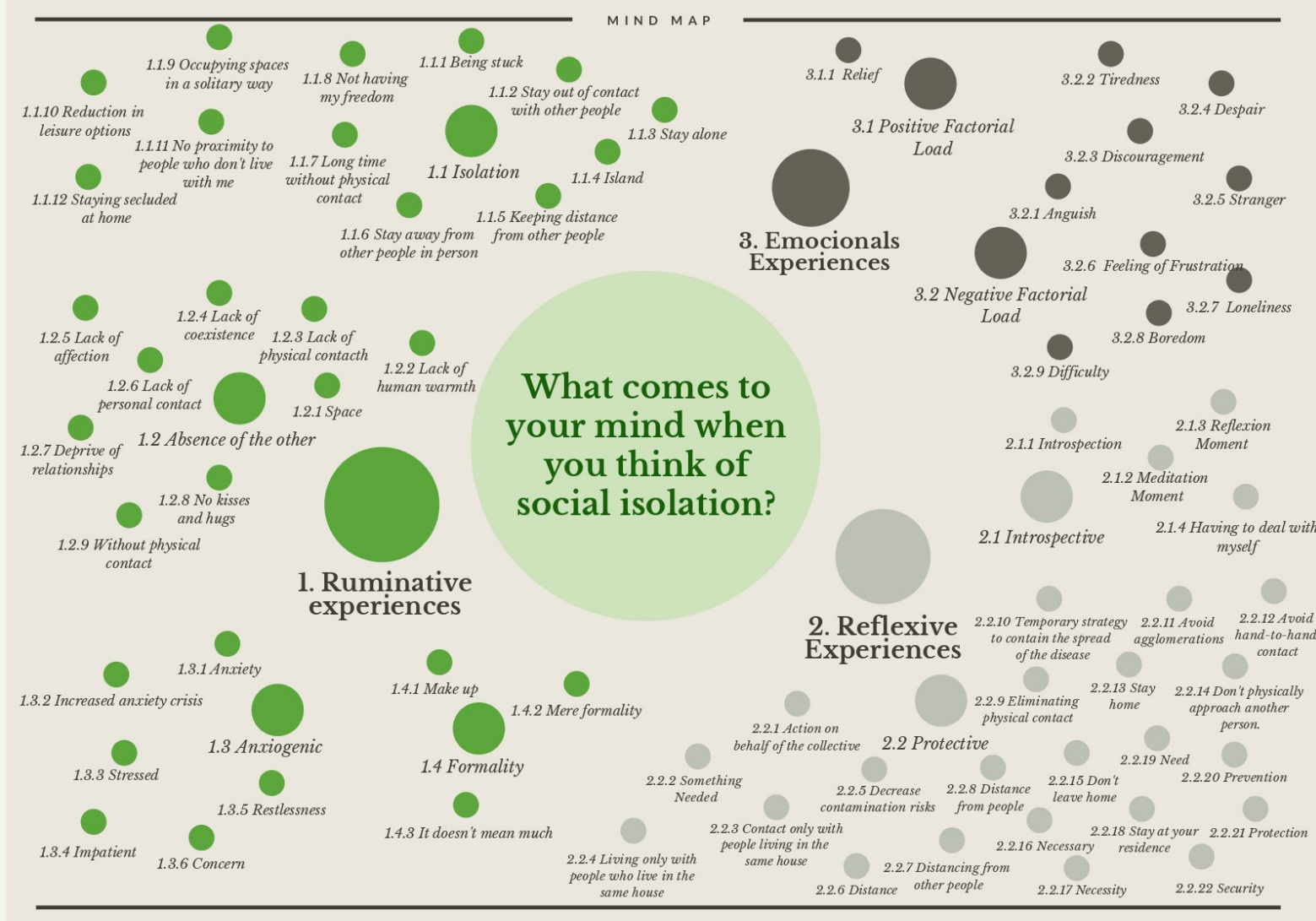

Figure 4: Representation of responses and categories found in the qualitative survey.

\section{Discussion}

Using self-report data collected through the Internet, the present work examined the relationship between loneliness and psychological distress at different levels of social isolation during the COVID-19 pandemic in Brazil, as well as how intrapersonal factors (coping strategies, external entrapment) affect this relationship. The relationship between media consumption of COVID-19-related information and psychological distress was also examined, as well as how coping strategies influence this relationship. We found support for the hypothesis that reliance on escape-avoidance and distancing coping strategies mediated the effects of social isolation-induced loneliness for psychological distress in the Brazilian population. However, we did not find support for the hypothesis that reliance on social support coping strategies significantly altered the effects of social isolation-induced loneliness on psychological distress. We also did not find support for the hypothesis that external entrapment moderated the effects of loneliness. We also found that the impact of 
reliance on positive reappraisal coping strategies on the relationship between frequency of media use for COVID-19-related information and psychological distress depended on the type of media, with individuals which sought information from print or online newspapers, social networks, and podcasts at higher frequencies consistently showing more psychological distress; however, higher levels of positive reappraisal coping strategies increased this impact instead of decreasing it. Interestingly, at the qualitative survey we also found that individuals interpreted isolation as producing self-assessment with protective and introspective dimensions, but also ruminative and emotional experiences of distress.

Loneliness has long been described as a factor that induces psychological distress, and as a result can be understood as a risk factor for many mental disorders ${ }^{14,15}$. If not accompanied by extensive policies on protecting mental health, community confinement strategies can lead to feelings of loneliness that can make managing stressful situations more difficult (i.e., can lead to non-adaptive coping strategies) and generate psychological distress ${ }^{5}$. The Brazilian response to the SARS-CoV2 pandemic, however, has been mixed, with social isolation being weakly enforced by government officials ${ }^{46,47}$. As a result, in Brazil social isolation became more a matter of individual practices than collective reasoning.

We found that, for individuals that reported more strict social isolation practices (i.e., individuals which reported leaving their houses less than once a week), feelings of loneliness were higher than for individuals that reported less strict social isolation practices, and this was accompanied by increased psychological distress. In fact, using SRQ-20, we found that $76.9 \%$ of the female respondents and $58.0 \%$ of the male respondents that reported leaving their houses less than once a week showed clinically significant symptoms (i.e., above the gender-defined cut-off points ${ }^{38}$ ), while these proportions fell below $65 \%$ for females and $44 \%$ for males that reported leaving their houses more than 3 times per week. Thus, not only was an increase in psychological distress observed, but this increase can be clinically relevant. These symptoms were also accompanied by feelings of anxiety related to illness, medical practices, and health-related routines, as evaluated by a scale for ambulatory patients ${ }^{39}$.

A sub-sample of participants also participated in Phase 2 of the research, responding to a cognitive free writing task in which they had to introspect about isolation. We found that these participants reported self-assessment of reflective character with protective and introspective dimensions; however, this reflective self-assessment was also articulated to ruminative and emotional experiences that were markedly tense, anxious and disparaging about self-scrutiny. The ruminative dimension is exemplary of feelings of loneliness and entrapment, in concordance with results from the quantitative survey.

In the present work, we found that relying on escape-avoidance and distancing 
coping strategies mediated the impacts of

Surprisingly, while social capital has been associated as a protective factor in the Chinese population at the beginning of the pandemic ${ }^{10}$, we did not find that reliance on social support coping strategies significantly altered the effects of social isolation-induced loneliness both SRQ-20 scores and illness anxiety in the Brazilian population.

We found that external entrapment was a significant moderator in the relationship between loneliness and SRQ-20, but not illness anxiety; however, we also found that this moderation did not change at any level of social isolation, suggesting that the sense of external entrapment is an important factor in loneliness-related psychological distress independent of self-isolation practices during the pandemic. A possible explanation for this lack of variation across levels is that external entrapment represents a feeling of lack of control ${ }^{21,23}$ that was also felt by individuals that were less isolated, as $83.9 \%$ of the individuals which reported leaving the house everyday did so because of occupational demands. Nonetheless, higher levels of entrapment weakened the relationship between loneliness and SRQ-20 scores, an effect that was opposite to what was hypothesized.

Morin ${ }^{48}$ argues that we spend much of our time analyzing our private thoughts and feelings, which shows the importance of observing how and how often we use introspection and can identify the effects on self-focus on our behavior. Self-awareness studies ${ }^{49-}$ 51 indicate that people generally engage in two types of self-analysis, one of a self-reflective character, which requires a purposeful assessment of the self, and another of self-ruminative character, which denotes a tense and anxious assessment of the self. Thus, in line with the current literature in the area, the findings of the present qualitative study reveal an emic repertoire of sense and significance of the subjective experience of physical isolation caused by the SARS-CoV2, characterized by a major presence of a reflective character selfassessment with protective and introspective dimensions; however, this reflective selfassessment was also articulated to ruminative and emotional experiences that were markedly tense, anxious and disparaging about the self's self-scrutiny. Overall, the results of the qualitative survey agree with the results from the quantitative survey in that they suggest psychologically distressing experiences and meanings, but also add that, for some individuals, these experiences can also represent meaningful moments to seek protection and to engage in self-care.

Our results reveal that social isolation during the COVID-19 pandemic significantly increased psychological distress at clinically relevant rates, with loneliness being an important predictor of this effect. We also found that escape-avoidance and distancing coping strategies mediated this effect. Psychological distress was also related to high 
consumption of COVID-19-related information in social networks, print or online newspapers, and podcasts, but that relying on positive reappraisal coping strategies increased this effect instead of decreasing it. These results suggest the need for policies that diminish the impact of social isolation on mental health; the need to assess and teach alternative coping strategies in clinical settings; and the need to address the impact of disinformation and perception of risk from Internet-based sources.

\section{References}

1. Bourouiba L. Turbulent Gas Clouds and Respiratory Pathogen Emissions. JAMA. March 2020. doi:10.1001/jama.2020.4756

2. Wilder-Smith A, Freedman DO. Isolation, quarantine, social distancing and community containment: pivotal role for old-style public health measures in the novel coronavirus (2019-nCoV) outbreak. J Travel Med. 2020;27:taaa020. doi:10.1093/jtm/taaa020

3. Anderson RM, Heesterbeek H, Klinkenberg D, Hollingsworth TD. How will countrybased mitigation measures influence the course of the COVID-19 epidemic? Lancet. 2020;395:931-934. doi:10.1016/S0140-6736(20)30567-5

4. van den Berge L. Biopolitics and the Coronavirus: Foucault, Agamben, Žižek. Netherlands J Leg Philos. 2020; No prelo.

5. Holmes EA, O'Connor RC, Perry VH, et al. Multidisciplinary research priorities for the COVID-19 pandemic: a call for action for mental health science. The Lancet Psychiatry. 2020;7:547-560. doi:10.1016/S2215-0366(20)30168-1

6. Qiu J, Shen B, Zhao M, Wang Z, Xie B, Xu Y. A nationwide survey of psychological distress among Chinese people in the COVID-19 epidemic: implications and policy recommendations. Gen Psychiatry. 2020;33:e100213. doi:10.1136/gpsych-2020100213

7. Pierce M, Hope H, Ford T, et al. Mental health before and during the COVID-19 pandemic: a longitudinal probability sample survey of the UK population. The Lancet Psychiatry. 2020;0366:1-10. doi:10.1016/s2215-0366(20)30308-4

8. Taquet M, Quoidbach J, Fried El, Goodwin GM. Mood Homeostasis Before and During the Coronavirus Disease 2019 (COVID-19) Lockdown Among Students in the 
Netherlands. JAMA Psychiatry. July 2020. doi:10.1001/jamapsychiatry.2020.2389

9. Portes A. Social Capital: Its Origins and Applications in Modern Sociology. Annu Rev Sociol. 1998;24:1-24. doi:10.1146/annurev.soc.24.1.1

10. Xiao H, Zhang Y, Kong D, Li S, Yang N. Social Capital and Sleep Quality in Individuals Who Self-Isolated for 14 Days During the Coronavirus Disease 2019 (COVID-19) Outbreak in January 2020 in China. Med Sci Monit. 2020;26:e923921-1e923921-8. doi:10.12659/MSM.923921

11. Mazza C, Ricci E, Biondi S, et al. A nationwide survey of psychological distress among Italian people during the covid-19 pandemic: Immediate psychological responses and associated factors. Int J Environ Res Public Health. 2020;17:1-14. doi:10.3390/ijerph17093165

12. Lau JTF, Yang X, Pang E, Tsui HY, Wong E, Wing YK. SARS-related Perceptions in Hong Kong. Emerg Infect Dis. 2005;11:417-424. doi:10.3201/eid1103.040675

13. Uchino BN, Cacioppo JT, Kiecolt-Glaser JK. The relationship between social support and physiological processes: A review with emphasis on underlying mechanisms and implications for health. Psychol Bull. 1996;119:488-531. doi:10.1037/00332909.119.3.488

14. Hawkley LC, Cacioppo JT. Loneliness and pathways to disease. Brain Behav Immun. 2003;17:98-105. doi:10.1016/S0889-1591(02)00073-9

15. Weiss RS. Loneliness: The Experience of Emotional and Social Isolation. Cambridge/ MA: MIT Press; 1973.

16. Cacioppo JT, Hawkley LC. Social Isolation and Health, with an Emphasis on Underlying Mechanisms. Perspect Biol Med. 2003;46:S39-S52. doi:10.1353/pbm.2003.0063

17. Wang P, Chen X, Gong J, Jacques-Tiura AJ. Reliability and Validity of the Personal Social Capital Scale 16 and Personal Social Capital Scale 8: Two Short Instruments for Survey Studies. Soc Indic Res. 2014;119:1133-1148. doi:10.1007/s11205-0130540-3

18. Bian M, Leung L. Linking Loneliness, Shyness, Smartphone Addiction Symptoms, and Patterns of Smartphone Use to Social Capital. Soc Sci Comput Rev. 2015;33:61-79. doi:10.1177/0894439314528779 
19. Cacioppo JT, Hawkley LC, Crawford LE, et al. Loneliness and Health: Potential Mechanisms. Psychosom Med. 2002;64:407-417. doi:10.1097/00006842-20020500000005

20. Russell D, Peplau LA, Ferguson ML. Developing a measure of loneliness. J Pers Assess. 1978;42:290-294.

21. Gilbert $P$, Allan $S$. The role of defeat and entrapment (arrested flight) in depression: An exploration of an evolutionary view. Psychol Med. 1998;28:585-598.

22. Maier SF, Amat J, Baratta M V., Paul E, Watkins LR. Behavioral control, the medial prefrontal cortex, and resilience. Dialogues Clin Neurosci. 2006;8(4):397-406. doi:10.1016/j.ynstr.2014.09.003

23. Gilbert P, Gilbert J. Entrapment and arrested fight and flight in depression: An exploration using focus groups. Psychol Psychother Theory, Res Pract. 2003;76:173188. doi:10.1348/147608303765951203

24. Carvalho S, Pinto-Gouveia J, Castilho P, Pimentel P. Entrapment - Conceito, definição e características psicométricas da versão portuguesa da Escala de Entrapment. Psychol - Avaliação Psicológica em Context Clínico. 2011;54:385-412.

25. Cloutier-Fisher D, Kobayashi K, Smith A. The subjective dimension of social isolation: A qualitative investigation of older adults' experiences in small social support networks. J Aging Stud. 2011;25:407-414. doi:10.1016/j.jaging.2011.03.012

26. Victor C, Scambler S, Bond J. The Social World Of Older People: Understanding Loneliness And Social Isolation In Later Life: Understanding Loneliness and Social Isolation in Later Life. Maidenhead: Open University Press/McGraw Hill Education; 2009.

27. Wenger GC, Davies R, Shahtahmasebi S, Scott A. Social Isolation and Loneliness in Old Age: Review and Model Refinement. Ageing Soc. 1996;16:333-358. doi:10.1017/ S0144686X00003457

28. Karnick PM. Feeling Lonely: Theoretical Perspectives. Nurs Sci Q. 2005;18:7-12. doi:10.1177/0894318404272483

29. Sell TK, Boddie C, McGinty EE, et al. Media Messages and Perception of Risk for Ebola Virus Infection, United States. Emerg Infect Dis. 2017;23:108-111. doi:10.3201/ eid2301.160589 
30. Wang Y, McKee M, Torbica A, Stuckler D. Systematic Literature Review on the Spread of Health-related Misinformation on Social Media. Soc Sci Med.

2019;240:112552. doi:10.1016/j.socscimed.2019.112552

31. Li S, Wang Y, Xue J, Zhao N, Zhu T. The Impact of COVID-19 Epidemic Declaration on Psychological Consequences: A Study on Active Weibo Users. Int J Environ Res Public Health. 2020;17:2032. doi:10.3390/ijerph17062032

32. Thompson RR, Garfin DR, Holman EA, Silver RC. Distress, Worry, and Functioning Following a Global Health Crisis: A National Study of Americans' Responses to Ebola. Clin Psychol Sci. 2017;5:513-521. doi:10.1177/2167702617692030

33. Thompson RR, Jones NM, Holman EA, Silver RC. Media exposure to mass violence events can fuel a cycle of distress. Sci Adv. 2019;5:eaav3502.

doi:10.1126/sciadv.aav3502

34. Bruner J. Acts of Meaning: Four Lectures on Mind and Culture. Cambridge/MA: Harvard University Press; 1993.

35. Couper MP. Review: Web surveys: A review of issues and approaches. Public Opin Q. 2000;64:464-494.

36. Schoemann AM, Boulton AJ, Short SD. Determining Power and Sample Size for Simple and Complex Mediation Models. Soc Psychol Personal Sci. 2017;8:379-386. doi:10.1177/1948550617715068

37. Tran V-T, Porcher R, Tran V-C, Ravaud P. Predicting data saturation in qualitative surveys with mathematical models from ecological research. $J$ Clin Epidemiol. 2017;82:71-78.e2. doi:10.1016/j.jclinepi.2016.10.001

38. Mari J, Williams P. A validity study of a psychiatric screening questionnaire (SRQ-20) in primary care in the city of São Paulo. Br J Psychiatry. 1986;148:23-28.

39. Oliveira JC da S, Sisto FF. Construção de uma escala de ansiedade para pacientes de ambulatório: um estudo exploratório. Psicol Teor e Prática. 2004;6:45-57.

40. Barroso SM, Andrade VS de, Midgett AH, Carvalho RGN de. Evidências de validade da Escala Brasileira de Solidão UCLA. J Bras Psiquiatr. 2016;65:68-75. doi:10.1590/0047-2085000000105

41. Savóia MG, Santana PR, Mejias NP. Adaptação do Inventário de Estratégias de Coping de Folkman e Lazarus para o português. Psicol USP. 1996;7:183-201. 
42. Kim DS, McCabe CJ, Yamasaki BL, Louie KA, King KM. Detecting random responders with infrequency scales using an error-balancing threshold. Behav Res Methods. 2018;50:1960-1970. doi:10.3758/s13428-017-0964-9

43. Baron RM, Kenny DA. The moderator-mediator variable distinction in social psychological research: Conceptual, strategic, and statistical considerations. J Pers Soc Psychol. 1986;51:1173-1182. doi:10.1037/0022-3514.51.6.1173

44. Gallucci M. jAMM: jamovi Advanced Mediation Models [jamovi module]. 2019.

45. Bardin L. Análise de Conteúdo. 3 ed. Lisbon: Edições 70; 2013.

46. The Lancet. COVID-19 in Brazil: "So what?" Lancet. 2020;395:1461. doi:10.1016/S0140-6736(20)31095-3

47. Zhu D, Mishra SR, Han X, Santo K. Social distancing in Latin America during the COVID-19 pandemic: an analysis using the Stringency Index and Google Community Mobility Reports. J Travel Med. 2020:taaa125. doi:10.1093/jtm/taaa125

48. Morin A. Do you "self-reflect" or "self-ruminate"? Sci Conscious Rev. 2002;1.

49. Fenigstein A, Scheier MF, Buss AH. Public and private self-consciousness: Assessment and theory. J Consult Clin Psychol. 1975;36:1241-1250. doi:10.1037/h0076760

50. Joireman JA, Parrott L, Hammersla J. Empathy and the self-absorption paradox: Support for the distinction between self-rumination and self-reflection. Self Identity. 2002;1:53-65. doi:10.1080/152988602317232803

51. Trapnell PD, Campbell JD. Private self-consciousness and the Five-Factor Model of personality: Distinguishing rumination from reflection. J Pers Soc Psychol. 1999;76:284-304. doi:10.1037/0022-3514.76.2.284 